\title{
Pleural MAC30 as a prognostic marker in NSCLC with malignant pleural effusion
}

\author{
Yi Shan ${ }^{1, *}$, Hui Ding ${ }^{2, *}$, Junjie Lu ${ }^{1}$, Zhijun Ge ${ }^{1}$ and Yongfei Tan ${ }^{3}$ \\ ${ }^{1}$ Department of Critical Care Medicine, The Affiliated Yixing Hospital of Jiangsu University Yixing, Jiangsu 214200, China \\ ${ }^{2}$ Department of Respiratory, The Affiliated Yixing Hospital of Jiangsu University Yixing, Jiangsu 214200, China \\ ${ }^{3}$ Department of Cardiac \& Thoracic Surgery, The Affiliated Yixing Hospital of Jiangsu University Yixing, Jiangsu 214200, \\ China \\ "These authors have contributed equally to this work
}

Correspondence to: Yongfei Tan, email: tan_yongfei@163.com

Keywords: MAC30; MPE; NSCLC; BPE; OS

Received: August 05, $2017 \quad$ Accepted: September 20, $2017 \quad$ Published: November 22, 2017

Copyright: Shan et al. This is an open-access article distributed under the terms of the Creative Commons Attribution License 3.0 (CC BY 3.0), which permits unrestricted use, distribution, and reproduction in any medium, provided the original author and source are credited.

\section{ABSTRACT}

Over-expressed meningioma-associate protein (MAC30) in tissues was associated with malignant tumor differentiation, metastasis and poor prognosis. However, the attention of MAC30 in pleural effusion from lung tumor is insufficient. Our retrospective study was prepared to explore the clinical values on diagnosis and prognosis of MAC30 from malignant pleural effusion (MPE) in non-small cell lung cancer (NSCLC). Levels of MAC30 were confirmed in MPE from 48 NSCLC patients and in benign pleural effusion (BPE) from 45 controls via enzyme-linked immunosorbent assay (ELISA). The association of MAC30 in MPE with clinical significance was further determined. We found that the levels of MAC30 in MPE were obviously higher than those in BPE $(p<0.05)$. Moreover, with a cutoff point $(17.5 \mathrm{ng} / \mathrm{ml})$, we confirmed the sensitivity and specificity of MAC30 for MPE were $82.7 \%$ and $85.3 \%$ using ROC curve analysis. Indeed, longer overall survival (OS) was present in NSCLC patients with low MAC30 expression in MPE. Multivariate analysis explicated that elevated MAC30 in MPE was an independent prognostic factor for shorter OS of NSCLC. Our data suggests that MAC30 in pleural effusion could be a potential prognostic marker in NSCLC with MPE.

\section{INTRODUCTION}

Lung malignancies stably control the leading cause of cancer-related death worldwide even with diagnostic and clinical therapeutic advances [1]. Accounting for more than $80 \%$ of lung cancers, non-small cell lung cancer (NSCLC) always shows metastases to distant sites at the time of diagnosis [2]. As a common clinical complication in more than $20 \%$ of NSCLC, Malignant Pleural Effusion (MPE) as an independent factor deteriorates the condition of patients and induces the poor survival [3]. Once the definition of MPE in NSCLC is confirmed, patients will be classified into advanced stages in TNM with a median survival of 5.5 months [4]. Thus, the distinction between MPE and benign pleural effusion (BPE) from cytological or histological diagnosis via thoracocentesis, pleural biopsy, and thoracoscopy is the first and critical step in clinical intervention in order to plan early initiation and suitable management. But, unfortunately, part of NSCLC patients with MPE always experience unclear diagnosis with cytological negative. Indeed, the detection of several tumor biomarkers such as carcinoembryonic antigen (CEA), cytokeratin 19 fragments (CYFRA 21-1) and carbohydrate antigen 153 (CA153) in pleural effusion is assisted to get proper diagnosis for patients with a deep suspicion of malignancy [5-7]. However, because of the unsatisfied diagnostic accuracy from 30 to $60 \%$, these biomarkers limit their clinical application value $[8,9]$. Therefore, it is essential to select an accurate biomarker for early detection of MPE and predicting prognosis of lung malignances.

Previous reports indicated that Meningiomaassociated protein (MAC30) locating on 17q11.2 [10] 
showed variously expression in different malignances, as over-expressed in breast, esophagus, lung and colon cancers [11-13] while down-expressed in pancreatic and renal cancers [14]. In deed, in our previous study, we already confirmed the elevated level of MAC30 in lung tissues from patient with NSCLC as a useful biomarker for poor prognosis and resistance to platinum-based chemotherapy $[13,15]$. However, it's unclear about the relationship of MAC in MPE with the clinical investigation in diagnosis and prognosis of NSCLC patients.

In this present study, we hypothesized that MA30 level was increased in NSCLC-induced pleural effusion. Moreover, we explored the clinical value of MAC30 in MPE on diagnostic and prognostic instructions.

\section{RESULTS}

\section{Pleural MAC30 expression in NSCLC}

The characteristics of a total of 48 NSCLC with MPE and 45 controls in the present study were summarized in Table 1. There's no statistical difference in age, gender and smoking history between the two groups. As shown in Figure 1, MAC30 level in MPE from NSCLC detected via ELISA was significantly increased than that in BPE $(18.25 \pm 6.11 \mathrm{ng} / \mathrm{ml}$ vs. $5.07 \pm 0.72 \mathrm{ng} / \mathrm{ml}, p<0.05)$ (Figure 1).

\section{Clinical diagnostic role of MAC30 on MPE}

In order to identify the clinical value of MAC in MPE, we prepared ROC curve with a cutoff point of MAC30 level $(17.5 \mathrm{ng} / \mathrm{ml})$ to ensure the diagnostic threshold that the area under the curve (AUC) was 0.891 (95\% CI 0.823-0.959) in Figure 2. Based on the judgment, MAC30 made a considerable sensitivity $82.7 \%$ and specificity $85.3 \%$.

\section{Pleural MAC30 level and clinicopathological elements in NSCLC with MPE}

The relationship between pleural MAC30 level and characteristics of NSCLC patients with MPE was shown in Table 2. As expected, MAC30 concentration in MPE was associated with distant metastasis, but not with age, gender, histological type and smoking. Furthermore, higher level of MAC30 in MPE was found in advanced NSCLC patients with metastasis to brain or bone.

\section{Prognostic value of MAC30 in MPE for advanced NSCLC patients}

According to the cutoff point $(17.5 \mathrm{ng} / \mathrm{ml})$, advance NSCLC patients with high expression of MAC30 in MPE were suffered with poorer OS via Kaplan-Meier curve analysis $(\mathrm{p}<0.05)$ (Figure 3$)$. Furthermore, the univariate and multivariate analyses indicated that MAC30 expression was an independent prognostic biomarker for OS in patients with NSCLC (Table 3). So, MAC30 expression in pleural effusion could act as a novel tumor biomarker for the prognosis of advanced NSCLC.

\section{DISCUSSION}

In the present study, we firstly identified the elevated levels of MAC30 in pleural effusion from advanced NSCLC compared with those in BPE controls. Further data confirmed that pleural MAC30 could discriminate lung cancer from BPE with considerable sensitivity and specificity for diagnostic rationality of MPE. Moreover, we not only confirmed the positive association between pleural effusion MAC30 and distant metastasis in NSCLC, but also found the poorer OS in patients with higher levels of pleural MAC30. To the best of our knowledge, we firstly demonstrated that pleural effusion MAC30 was

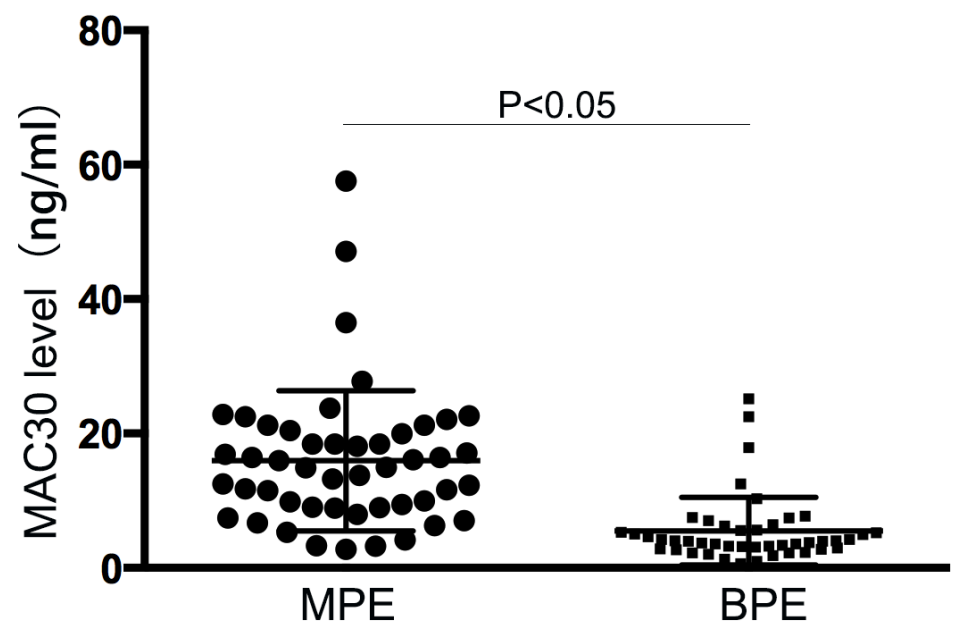

Figure 1: The levels of MAC30 in pleural effusion. Elevated MAC30 levels in MPE was presented than those in BPE $(p<0.05)$. 
Table 1: Various clinicopathological features of patients

\begin{tabular}{|c|c|c|c|}
\hline Variables & MPE & BPE & $p$ value \\
\hline No. & 48 & 45 & \\
\hline Age (years) & & & 0.773 \\
\hline$<59$ & 31 & 26 & \\
\hline$\geq 59$ & 17 & 19 & \\
\hline Gender & & & 0.586 \\
\hline Male & 26 & 21 & \\
\hline Female & 22 & 24 & \\
\hline Smoking status & & & 0.612 \\
\hline Non-smoker & 31 & 28 & \\
\hline Smoker & 17 & 17 & \\
\hline \multicolumn{4}{|l|}{ Histological type } \\
\hline SQCLC & 11 & ND & \\
\hline $\mathrm{AC}$ & 37 & ND & \\
\hline AS & 0 & ND & \\
\hline \multicolumn{4}{|l|}{$\mathrm{BPE}$} \\
\hline Tuberculous & ND & 9 & \\
\hline Parapneumonic & ND & 28 & \\
\hline Others & ND & 8 & \\
\hline
\end{tabular}

MPE, malignant pleural effusion; SQCLC, squamous cell carcinomas; AC, adenocarcinoma; AS, adenosquamous; BPE, benign pleural effusion; ND, no data;

an independent prognostic marker for OS of advanced NSCLC. So, our findings imply that MAC30 in pleural effusion could act as a potential diagnostic marker for lung cancer-associated malignant effusion.



Figure 2: Diagnostic value of pleural fluid MAC30 levels for NSCLC patients with MPE. Based on a cutoff value of 17.5 $\mathrm{ng} / \mathrm{ml}$ according to pleural fluid MAC30 concentrations (AUC $=0.891 ; 95 \% \mathrm{CI}=0.823-0.959)$, the ROC curve discrimination of MPE and BPE presents the sensitivity $82.7 \%$ and specificity $85.3 \%$. 
Table 2: MAC30 in MPE with the clinicopathological factors of NSCLC patients

\begin{tabular}{lccc}
\hline Variables & Number & MAC30 (ng/ml) & $p$ value \\
\hline Age (years) & 31 & & 0.518 \\
$\quad<59$ & 17 & 13.556 & \\
$\geq 59$ & & & 0.275 \\
Gender & 26 & 15.648 & \\
$\quad$ Male & 22 & 16.994 & 0.887 \\
Female & & & \\
Smoking status & 31 & 15.937 & 0.665 \\
$\quad$ Non-smoker & 17 & 13.728 & \\
Smoker & & & \\
Histological type & 11 & 18.664 & $\mathbf{0 . 0 1 3}$ \\
SQCLC & 37 & 15.832 & \\
AC & & & \\
Distant metastasis & 22 & 12.446 & \\
Absent & 26 & & \\
Present & & & \\
\hline
\end{tabular}

SQCLC, squamous cell carcinomas; AC, adenocarcinoma;

The diagnostic use of biomarkers for MPE is based on discriminating lung cancer from BPE with considerable sensitivity and specificity. Previous studies have already investigated the clinical value of several biomarkers in pleural fluid including (CEA), CYFRA21-1, CA125 and Romo1 [16-18]. Among those, CEA, CYFRA21-1 and CA125 were widely accepted in clinical performance as to distinguishing MPE from BPE. However, the real availability in clinical diagnostic of malignancies is doubtful. As a glycoprotein component of glycocalyx in the endothermic epithelium, CEA was confirmed to act as an biomarker for the diagnosis of MPE with a sensitivity from $29 \%$ to $82 \%$ and a specificity ranging from $77 \%$ to $93 \%$ [5]. Indeed, the increased expression of CEA in pleural fluid also could be found in nonmalignant diseases, as inflammatory, lung tuberculosis and pulmonary fibrosis. CYFRA21-1 expressing especially in lung squamous cell carcinoma [17] yielded sensitivity from $22 \%$ to $91 \%$ and specificity from $80 \%$ to $97 \%$ [19]. Moreover, the previous study revealed that there was

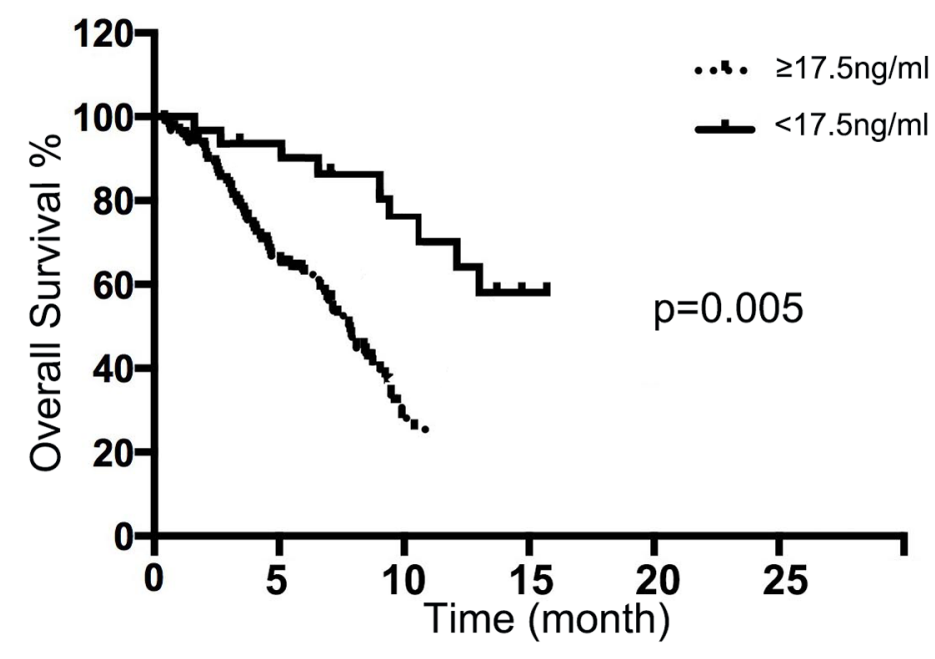

Figure 3: Pleural fluid MAC30 expression with overall survival of NSCLC patients. The NSCLC patients with high MAC30 expression had a significantly worse outcome compared with the patients with low MAC30 level $(p=0.005)$. 
Table 3: Univariate and multivariate analysis of prognostic factors in NSLCL patients

\begin{tabular}{lcccc}
\hline Variables & \multicolumn{2}{c}{ Univariate } & \multicolumn{2}{c}{ Multivariate } \\
\cline { 2 - 5 } & HR & P value & HR & P value \\
\hline MAC30 expression & 1.373 & $\mathbf{0 . 0 1 2}$ & $\mathbf{0 . 0 0 8}$ \\
Age (years) & 1.736 & 0.551 & 0.484 & \\
Gender & 1.198 & 0.582 & & \\
Histological type & 1.331 & 0.433 & 1.482 & $\mathbf{0 . 0 0 5}$ \\
Smoking status & 1.236 & $\mathbf{0 . 0 0 7}$ & \\
Distant metastasis & 1.177 & & \\
\hline
\end{tabular}

little clinical difference in identifying lung caner-related MPE between CEA and CYFRA21-1 [20]. A recent report suggested that concurrent use of pleural fluid CEA and Romo1 showed better diagnostic performance than did CEA or CYRFA 21-1 alone [17], but the sample in the study was relatively small. In this study, we found that higher level of MAC30 in pleural effusion presented an acceptable sensitivity and specificity for the diagnosis of MPE. In addition, pleural MAC30 associating with OS was an independent biomarker for prognosis of NSCLC with MPE. The clinical use of MAC30 as a promising noninvasive biomarker for NSCLC diagnosis and prognosis was viewed.

MAC30, as a member of the insulin-like growth factor-binding protein family (IGFBP), was approved to play as a suppressor or a promoter in different cancers. As a newly concerned gene, the biological influence of MAC30 in tumor metabolism remains unclear. Pervious study confirmed that enhanced MAC30 expression in oral squamous cell carcinoma was positive with lymph nodal metastasis and server clinical prognosis [21]. Indeed, our previous studies confirmed that NSCLC patients with higher MAC30 expression resisted to platinum-based chemotherapy and exhibited worse survival $[13,15]$. It's difficult for NSCLC patients with pleural effusion to receive invasive procedures including needle pleural biopsy or thoracostomy in order to get cytological examination. Meanwhile, it's critical to clear that the pleural effusion in NSCLC is MPE or BPE, because MPE not only indicates advanced disease but also comes with poor prognosis. In clinical process, a lung cancer patient with pleural effusion is firstly classified into advance cancer stage. However, Porcel et al [22] demonstrated that pleural effusions in NSCLC also could be benignant especially combining with obstructive pneumonia or heart failure. Biomarker in pleural effusion was investigated to identify its nature associating with the progress of disease. However, the clinical use is limited in most markers [3]. Based on our previous findings in lung tissue, we hypothesized that the expression of MAC30 in MPE was elevated compared with that in BPE. To the best of our knowledge, our data firstly indicated that pleural fluid
MAC30 was contributed to determine whether the effusion is malignant or benignant. Thus, it is clinically meaningful to select sensitive markers for malignant effusion.

The present study verified that MAC30 in pleural effusions presented as a clinical prognostic marker of OS in advanced NSCLC. However, the study also contains several limitations. First, the collected size was relatively small because of a retrospective study. The further step is to enroll more samples to confirm our points. Second, the paired serum samples were lacking. The both measurement of serum and pleural fluid MAC30 could provide us more information about the sensitive and reliable expression of MAC30 in determining pleural fluid etiology. Third, although we confirmed that MAC30 expression in lung tissue was related to both poor response and prognosis in NSCLC with platinum-based chemotherapy, the possible relationship between pleural fluid MAC30 with therapeutic response was not confirmed. Thus, further research is focused on the conclusion whether MAC30 in MPE could be a predictive factor for platinum-based therapy response.

In conclusion, our present data suggested that pleural MAC30 concentration showed acceptable diagnostic sensitivity and accuracy to predict whether a pleural effusion is benign or malignant. Moreover, the levels of MAC30 in MPE could reflect the prognosis of NSCLC. In addition, our study provided clues for future observation about the clinical implication of MAC30 in NSCLC with MPE.

\section{MATERIALS AND METHODS}

\section{Patients and tissue samples}

In our study, we prospectively enrolled 48 NSCLC patients with MPE who were admitted to the department of Respiratory and Critical Care Medicine at Yixing people hospital affiliated Jiangsu University from May 2011 and March 2016. And 45 BPE from non-cancer patients were collected as controls in our study. Patients who had received preoperative chemotherapy were excluded. The present study was approved by the ethics committees of 
Jiangsu University Faculty of Medicine. The informed consent obtained from all subjects was confirmed.

\section{Study subjects and specimens}

Pleural effusion was collected from subjects and centrifuged at $3000 \mathrm{rpm}$ for $10 \mathrm{~min}$ at $4^{\circ} \mathrm{C}$, and then supernatant was frozen and stored at $-80^{\circ} \mathrm{C}$ until analysis. According to the manufacturer's instructions of ELISA kit (Mlbio, Shanhai, China), we determined MAC30 expression in pleural effusion. All samples were checked in duplicates.

\section{Statistical analysis}

All data was analyzed by SPSS version 17.0. The level of statistical significance was considered as $p<0.05$ for all tests. The diagnostic value of $\mathrm{MAC} 3 \mathrm{O}$ was assessed with ROC curve. Moreover, overall survival (OS) was performed by Kaplan-Meier analysis following with the relationship with MAC30 levels. Several factors as age, gender, histological type, smoking index, histological type and MAC30 were assessed to confirm the significance for prognosis via univariable and multivariate Cox. Independent prognostic factors influencing OS were identified via a cox regression model.

\section{CONFLICTS OF INTEREST}

The authors report no conflicts of interest in this work.

\section{FUNDING}

Grant (QNRC2016210) from the Science Foundation of Health Department of Jiangsu Province in China and Grant (81500049) from National Natural Science Foundation of China and supported this study.

\section{REFERENCES}

1. Siegel RL, Miller KD, Jemal A. Cancer Statistics. CA Cancer J Clin. 2017; 67:7-30.

2. Morgensztern D, Waqar S, Subramanian J, Trinkaus K, Govindan R. Prognostic impact of malignant pleural effusion at presentation in patients with metastatic nonsmall-cell lung cancer. J Thorac Oncol. 2012; 7:1485-9.

3. Porcel JM, Gasol A, Bielsa S, Bielsa S, Civit C, Light RW, Salud A. Clinical features and survival of lung cancer patients with pleural effusions. Respiratory. 2015; 20:654-9.

4. William WN Jr, Lin HY, Lee JJ, Lippman SM, Roth JA, Kim ES. Revisiting stage IIIB and IV non-small cell lung cancer: analysis of the surveillance, epidemiology, and end results data. Chest. 2009; 136:701-9.

5. Lee JH, Chang JH. Diagnostic utility of serum and pleural fluid carcinoembryonic antigen, neuron-specific enolase, and cytokeratin 19 fragments in patients with effusions from primary lung cancer. Chest. 2005; 128:2298-303.

6. Shi HZ, Liang QL, Jiang J, Qin XJ, Yang HB. Diagnostic value of carcinoembryonic antigen in malignant pleural effusion: a meta-analysis. Respiratory. 2008; 13:518-27.

7. Wu Q, Li M, Zhang S, Chen L, Gu X, Xu F. Clinical diagnostic utility of CA 15-3 for the diagnosis of malignant pleural effusion: a meta-analysis. Exp Ther Med. 2015; 9:232-38.

8. Nguyen AH, Miller EJ, Wichman CS, Berim IG, Agrawal DK. Diagnostic value of tumor antigens in malignant pleural effusion: a meta-analysis. Transi Res. 2015; 166:432-9.

9. Sriram KB, Relan V, Clarke BE, Duhig EE, Yang IA, Bowman RV, Lee YC, Fong KM. Diagnostic molecular biomarkers for malignant pleural effusions. Future Oncol. 2011; 7:737-52.

10. Murphy M, Pykett MJ, Harnish P, Zang KD, George DL. Identification and characterization of genes differentially expressed in meningiomas. Cell Growth Differ. 1993; 4:715-22.

11. Xiao M, Li H, Yang S, Huang Y, Jia S, Wang H, Wang J, Li Z. Expression of MAC30 protein is related to survival and clinicopathological variables in breast cancer. J Surg Oncol. 2013; 107:456-62.

12. Moparthi SB, Arbman G, Wallin A, Kayed H, Kleeff J, Zentgraf H, Sun XF. Expression of MAC30 protein is related to survival and biological variables in primary and metastatic colorectal cancers. Int J Oncol. 2007; 30:91-5.

13. Chen R, Fen Y, Lin X, Ma T, Cai H, Ding H. Overexpression of MAC30 is resistant to platinum-based chemotherapy in patients with non-small cell lung cancer. Technol Cancer Res Treat. 2016; 15:815-20.

14. Kayed H, Kleeff J, Ding J, Hammer J, Giese T, Zentgraf H, Buchler MW, Friess H. Expression analysis of MAC30 in human pancreatic cancer and tumors of the gastrointestinal tract. Histol Histopathol. 2004; 19:1021-31.

15. Ding H, Gui X, Lin X, Chen R, Ma T, Sheng Y, Cai H, Fen Y. The prognostic effect of MAC30 expression on patients with non-small cell lung cancer receiving adjuvant chemotherapy. Technol Cancer Res Treat. 2017; 16:645-53.

16. Ferrer J, Villarino MA, Encabo G, Felip E, Bermejo B, Vila S, Orriols R. Diagnostic utility of CYFRA 21-1, carcinoembryonic antigen, CA 125, neuron specific enolase, and squamous cell antigen level determinations in the serum and pleural fluid of patients with pleural effusions. Cancer. 1999; 86:1488-95.

17. Lai RS, Chen CC, Lee PC, Lu JY. Evaluation of cytokeratin 19 fragment (CYFRA 21-1) as a tumor marker in malignant pleural effusion. Jpn J Clin Oncol. 1999; 29:421-4.

18. Lee SH, Park MJ, Choi SI, Lee EJ, Lee SY, In KH. Reactive oxygen species modulator 1 (Romo1) as a novel diagnostic marker for lung cancer-related malignant effusion. Medicince. 2017. 96:e5975. 
19. Alatas F, Alatas O, Metintas M, Colak O Harmanci E, Demir S. Diagnostic value of CEA, CA 15-3, CA 19-9, CYFRA 21-1, NSE and TSA assay in pleural effusions. Lung Cancer. 2001; 31:9-16.

20. Gu P, Huang G, Chen Y, Zhu C, Yuan J, Sheng S. Diagnostic utility of pleural fluid carcinoembryonic antigen and CYFRA 21-1 in patients with pleural effusion: a systematic review and meta-analysis. J Clin Lab Anal. 2007; 21:398-405.
21. Yan BY, Wang DW, Zhu ZL, Yang YH, Wang MW, Cui DS, Zhang H, Sun XF. Overexpression of MAC30 in the cytoplasm of oral squamous cell carcinoma predicts nodal metastasis and poor differentiation. Chemotherapy. 2010; 56: 424-8.

22. Porcel JM, Azzopardi M, Koegelenberg CF, Maldonado F, Rahman NM, Lee YC. The diagnosis of pleural effusions. Expert Rev Respir Med. 2015; 9:801-15. 